\title{
Evolution of the initially ordered structure in a magnetic fluid film during a thermal cycle
}

\author{
Y.H. Ke ${ }^{1}$, S.Y. Yang ${ }^{2}$, H.E. Horng ${ }^{1,2, a}$, Chin-Yih Hong ${ }^{3}$, and H.C. Yang ${ }^{4}$ \\ 1 Department of Physics, National Taiwan Normal University, Taipei 116, Taiwan \\ 2 Institute of Electro-optical Science and Technology, National Taiwan Normal University, Taipei 116, Taiwan \\ 3 Department of Mechanical and Automation Engineering, Da-Yeh University, Changhwa 515, Taiwan \\ 4 Department of Physics, National Taiwan University, Taipei 106, Taiwan
}

Received 1st December 2003 / Received in final form 28 August 2004

Published online 21 October 2004 - (C) EDP Sciences, Società Italiana di Fisica, Springer-Verlag 2004

\begin{abstract}
A hexagonal ordered structure of magnetic columns, which results from an agglomeration of magnetic particles, is obtained in a magnetic fluid film when a magnetic field is applied perpendicularly to the film surface. The evolution of the initially ordered structure in the magnetic fluid film during the heating and cooling process is investigated under a given magnetic field. For the heating process, the columns remain unchanged until the temperature exceeds a critical temperature. As the temperature is further increased, column particles start to disperse into the liquid carrier. As a result, portions of columns disappear. As the temperature continue to rise, the ordered structure changes to a disordered column state, or even a monodispersed state. On the other hand, when the temperature is lowered, the magnetic particles in the carrier condense out of solution and finally an ordered structure of columns is achieved. However, this structural evolution during a thermal cycle is irreversible.
\end{abstract}

PACS. 75.50.Mm Magnetic liquids - 64.75.+g Solubility, segregation, and mixing; phase separation 68.60.Dv Thermal stability; thermal effects

\section{Introduction}

With the agglomeration of magnetic nano-particles, a variety of pattern-forming systems have been observed in magnetic fluid films under external magnetic fields [1-9]. Among these structural patterns, the most particular one may be the ordered hexagonal structure of magnetic columns in highly homogeneous magnetic fluid films under magnetic fields perpendicular to the films $[5,9]$. This ordered structure in magnetic fluid film can diffract an incident light to generate significant magnetochromatics $[10,11]$, thus exhibiting great potential for application in optical switches, displays, gratings etc. [12]. Further investigation has shown that this ordered structure can be manipulated by adjusting the magnetic field strength, the sweep rate of the field, the film thickness, the concentration of the magnetic particles in fluid [13], etc. These results reveal the feasibility of developing dynamic or tunable optical devices by utilizing the tunable ordered structures in magnetic fluid film under perpendicular magnetic fields [14].

Practically, heat consumption usually occurs when a device is under operation. This leads to a variation in

\footnotetext{
a e-mail: phyfv001@scc.ntnu.edu.tw
}

temperature of the device, and in turn may disturb the ordered structure in the magnetic fluid film. However, the influence of thermal variation in the ordered structure is still unclear, and hence the thermally activated change in optical properties generated by the ordered structure is also undetermined. In this work, we investigate in detail the structural evolution of the ordered structure in the magnetic fluid film as the temperature rises to a certain value and then drops down to the initial value.

\section{Experimental details}

The kerosene-based $\mathrm{MnFe}_{2} \mathrm{O}_{4}$ magnetic fluid with saturated magnetization of $7.7 \mathrm{emu} / \mathrm{g}$ was injected to a $6 \mu \mathrm{m}$-thick glass cell to form a magnetic fluid thin film. This film was positioned inside a vacuum cell, whose temperature was controlled with a circulating water system. A scheme to illustrate the temperature-control cell is shown in Figure 1. A LakeShore Pt-111 temperature sensor, which was located beside the film, was used to monitor the temperature to within $0.1^{\circ} \mathrm{C}$. Then, the film, together with the cell, were subjected to a pair of solenoids, which generated a uniform magnetic field perpendicular to the 

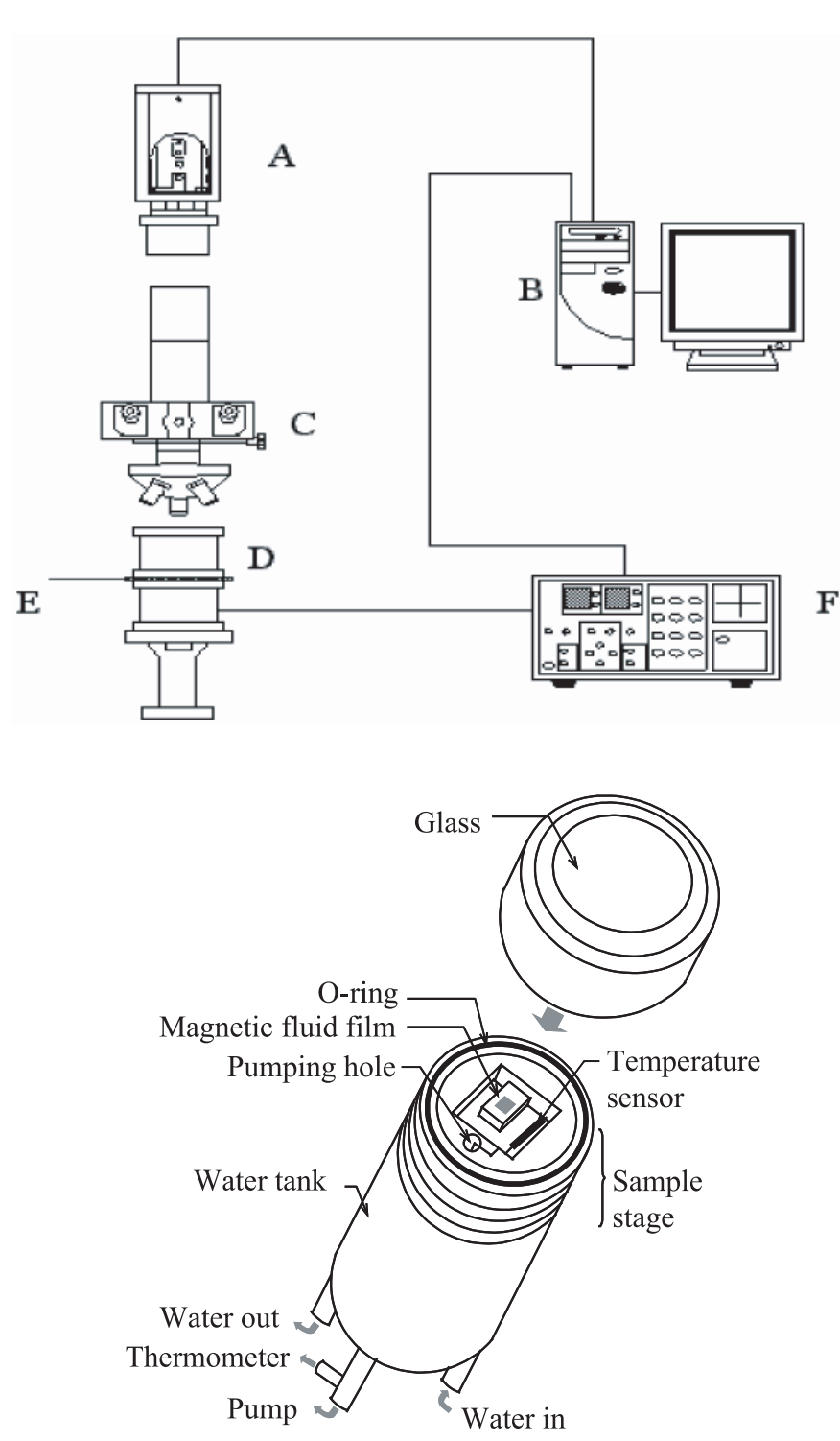

Fig. 1. Scheme of the temperature-controlling cell. The water tank is made of stainless, and the sample stage is made of cooper.

plane of the film. To obtain an ordered structure in the film, a magnetic field was applied to the film, increasing from zero to the desired strength at a given sweep rate $(=5 \mathrm{Oe} / \mathrm{s})$ and a fixed temperature $\left(=26.0^{\circ} \mathrm{C}\right.$ here $)$. Hereafter, the field was fixed at the desired strength. After the structure reached equilibrium, the ordered structure was recorded with the aid of a CCD camera and an optical microscope. We then increased the temperature gradually to $27.0^{\circ} \mathrm{C}$, at which point the temperature was hold for 15 minutes so that the structure was able to reach to an equilibrium state at $27.0{ }^{\circ} \mathrm{C}$. The temperature was then increased in this way to a final temperature, and lowered in steps down to the initial temperature. Thus, the structural evolution during a heating/cooling process under a given field strength was able to be investigated.

\section{Result and discussion}

To investigate the evolution of an ordered structure in the magnetic fluid film during a heating process, an external field was increased to a certain strength, here 120 Oe, at $26.0{ }^{\circ} \mathrm{C}$ to obtain the initially-ordered structure. An image of the structural pattern at equilibrium is shown in Figure 2a. As the temperature increased, the structural pattern remained almost unchanged until the temperature exceeded a critical temperature $\left(=35.0{ }^{\circ} \mathrm{C}\right.$ here $)$ at which point the column particles started to disperse into the liquid carrier, and number of columns reduced. The unchanged structural pattern at temperatures from 26.0 to $35.0{ }^{\circ} \mathrm{C}$ indicates that the equilibrium energy of the ordered structure in the magnetic fluid film exhibits a potential well with varying temperature. Moreover, this structure does not change until the excess kinetic energy of magnetic particles is over the potential well at higher temperatures. A similar phenomenon was observed for the structural evolution in magnetic fluid films under increasing magnetic field [9], where the potential well results from the long-range grain boundaries in the ordered structure. When the temperature rises from $35.0^{\circ} \mathrm{C}$, more columns disappear and the interaction between magnetic columns is reduced. As the temperature reaches $40.0^{\circ} \mathrm{C}$, the column-column interaction nearly vanishes and hence the structural pattern evolves to a disordered state, as shown in Figure 2c. This phenomenon is similar to the two-dimensional melting process observed for magnetic bubble arrays in garnet films [15] and is usually interpreted based on the theory proposed by Halperin and Nelson [16]. At temperatures higher than $56.0^{\circ} \mathrm{C}$, the influence of the thermal energy completely depresses that of magnetic energy on magnetic particles. As a result, all the originally agglomerated magnetic particles are dispersed into the liquid, as shown in Figure 2d, and the structure became monodispersed.

As to the structural evolution during a cooling process, the dispersed particles started to agglomerate to form magnetic columns when the temperature was reduced to $54.0^{\circ} \mathrm{C}$. New columns continued to form as the temperature was reduced to $51.0^{\circ} \mathrm{C}$. From 51.0 to $41.0{ }^{\circ} \mathrm{C}$, the dispersed magnetic particles condensed to existing columns. Thus, the number of columns remained unchanged, but each column became larger with as the temperature reduced. When the temperature was reduced below $41.0^{\circ} \mathrm{C}$, the columns were so large that the surface tension of column could not confine the column particles. Consequently, each column split into two columns. According to experimental observation, the total volume of the two new columns is larger than that of the original column. This implies that partially dispersed particles agglomerated with the new columns when the split occurred. This process continued as the temperature was lowered to $26{ }^{\circ} \mathrm{C}$, and meanwhile, the number of columns increased, as shown in Figures 2e and f. With the increase in number of columns, the interaction between magnetic columns was enhanced. This led to re-formation of the ordered structure in the magnetic fluid film when the temperature reduced $29.0{ }^{\circ} \mathrm{C}$. Figure $2 \mathrm{~g}$ gives the ordered 
(a) $\mathrm{T}=26^{\circ} \mathrm{C}$

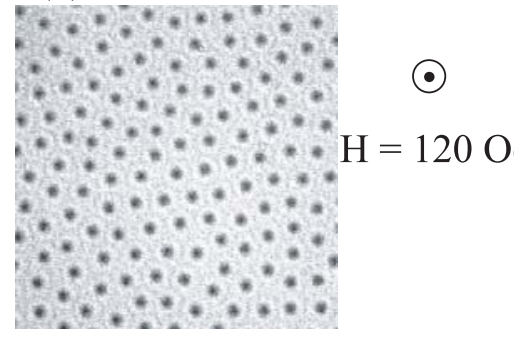

(b) $\mathrm{T}=32{ }^{\circ} \mathrm{C}$

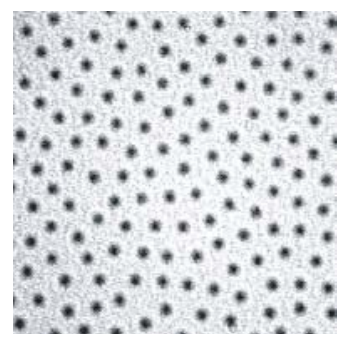

(c) $\mathrm{T}=40{ }^{\circ} \mathrm{C}$

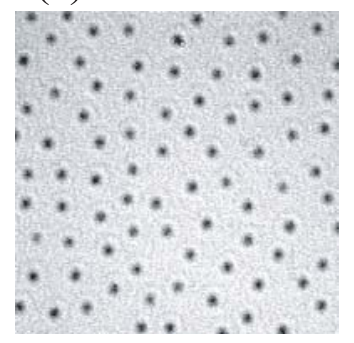

(d) $\mathrm{T}=56^{\circ} \mathrm{C}$

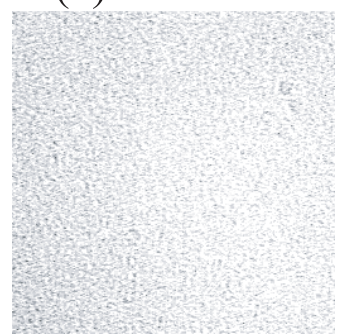

Fig. 2. Structural evolution of the initially ordered pattern in the magnetic fluid film under 120 Oe for $(\mathrm{a}-\mathrm{d})$ the heating process and then $(\mathrm{e}-\mathrm{g})$ the cooling process. The final structural state of the heating process is monodispersed.

structure at $26.0{ }^{\circ} \mathrm{C}$ after the cooling process. Remarkably, although the structural patterns in Figures 2a and $\mathrm{g}$ are ordered, the period (columns spacing) of the ordered structure has become larger after the thermal cycle. This result means that the change in structural pattern is irreversible after a thermal cycle.

According to our previous studies [9], the column formation deeply depends on the sweep rate of the applied magnetic field; the higher the sweep rate, the smaller the column diameter. Hence, the ordered structures formed with columns are dominated by the sweep rate. The thermal energy of particles shows an opposite effect on the column formation as compared to the application of a mag- netic field. Thus, in this work, when the temperature is lowered from 56.0 to $26.0^{\circ} \mathrm{C}$ to make particles agglomerated, it is effective to the case in which a magnetic field is applied to the magnetic fluid film at a certain rate. Since the reduction in the temperature is so slow that the effective sweep rate is smaller than that $(5 \mathrm{Oe} / \mathrm{s})$ at which the magnetic field is applied to 120 Oe to achieve the initial ordered structure shown in Figure 2a, the column diameter observed in Figure $2 \mathrm{~g}$ is larger than that in Figure 2a. And hence, an irreversible behavior is resulted for the ordered structure in a magnetic fluid film during a thermal cycle.

To qualitatively describe the structural evolution during a thermal cycle, the structures shown in Figure 2 are analyzed. Since the structure evolves through several states during a thermal cycle, various methods are adopted to characterize these structural states. First of all, for the initially ordered structure, we located each column in Figure 2a and then calculated the spacing between two nearest neighboring columns. The inset in Figure 3a displays the distribution of column spacing at $26{ }^{\circ} \mathrm{C}$. It was found that the distribution of column spacing $\rho(s)$ follows a Gaussian distribution

$$
\rho(s)=\frac{b}{\sigma \sqrt{\pi / 2}} \exp \left[-2 \frac{(s-d)^{2}}{\sigma^{2}}\right]
$$

with an average value, $d$, of $3.85 \mu \mathrm{m}$, and a standard deviation, $\sigma$, of $0.75 \mu \mathrm{m}$ and $b$ being 0.054 for the ordered structure shown in Figure 2a. The good consistence between the data points and the Gaussian curve shown in the inset in Figure 3a implies that the system follows a behavior of classical probability in entity. This may be due to that the dimension of the columns or the column spacing is the order of magnitude around micrometers. For this dimension scale, the system should exhibit classical, instead of quantum, statistic properties.

As mentioned above, the structural pattern remained unchanged as the temperature rose from 26.0 to $35.0{ }^{\circ} \mathrm{C}$. Hence, a constant value resulted for the average column spacing $d$ of the ordered structure in the magnetic fluid film as the temperature increased from 26.0 to $35.0^{\circ} \mathrm{C}$, as shown in Figure 3a. This was followed by an increase in the value of $d$ at temperatures higher than $35.0{ }^{\circ} \mathrm{C}$ due to the dispersion of magnetic columns. When the temperature exceeds $40.0{ }^{\circ} \mathrm{C}$, the structure evolved to a disordered column state, at which the average column spacing became insignificant and was not analyzed. Before qualitatively analyzing the structural evolution at the disordered states with varying temperatures higher than $40.0^{\circ} \mathrm{C}$, another feature, orderness $Q$, of the ordered structures was also investigated.

The orderness of the ordered structure can be calculated via $Q=d / \sigma$, where $d$ and $\sigma$ are obtained by fitting the experimental $\rho(s)$ to equation (1). For example, the $Q$ for the ordered structure shown in Figure 2a was obtained as 5.13. By definition, the $Q$ means the degree of dispersion in values of the column spacing in an ordered structural pattern. The larger the $Q$, the smaller the dispersion of column spacing. For an asymptotic case in which $Q$ is 


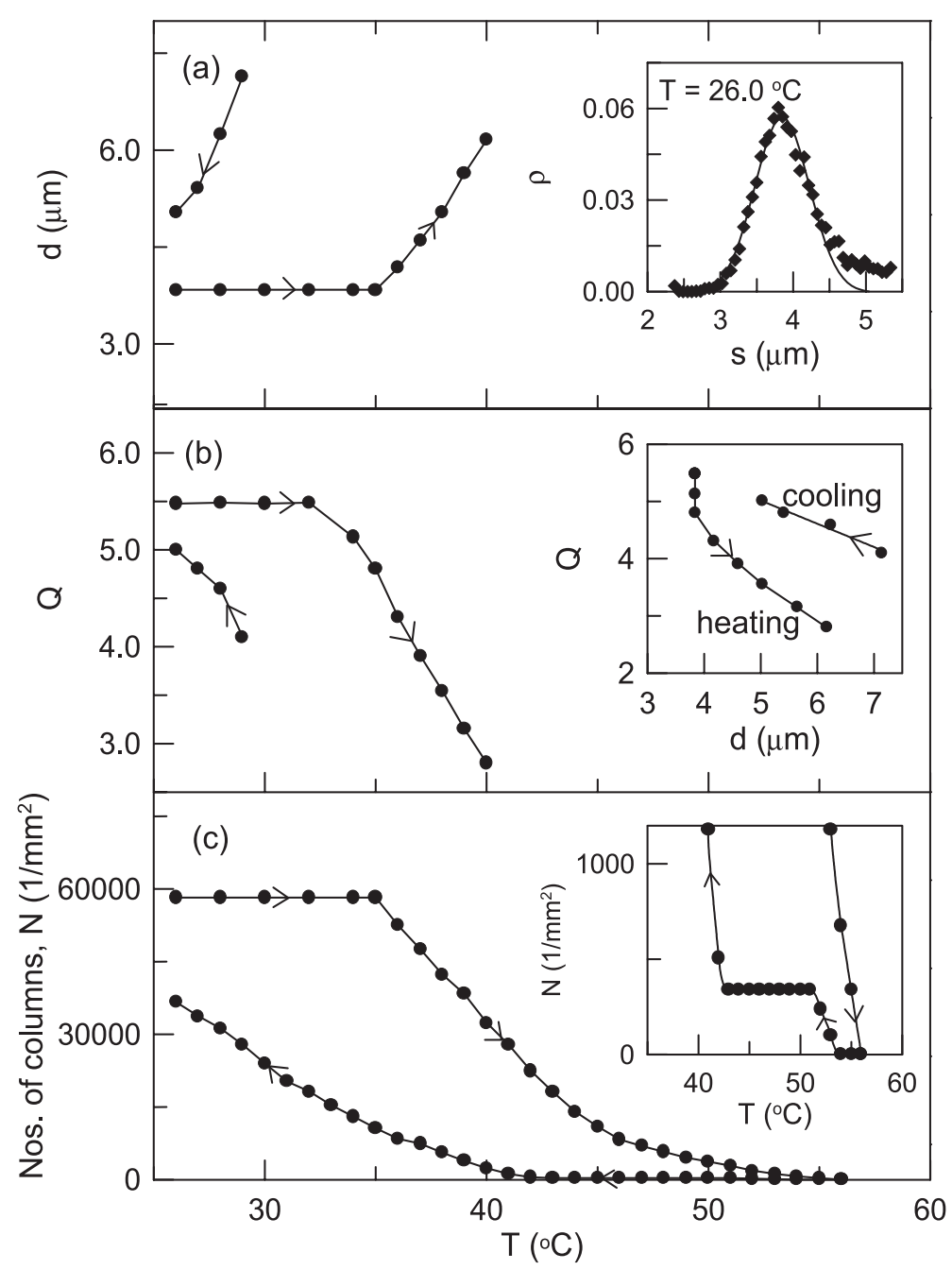

Fig. 3. (a) Column spacing, (b) orderness, and (c) column number within per unit area in the magnetic fluid film under 120 Oe for the thermal cycle with the final temperatures being $56.0^{\circ} \mathrm{C}$ for the heating process. The inset in (a) shows the Gaussian distribution for the column spacing of the ordered structure in the magnetic fluid film. The inset in (b) plots the column spacing dependent orderness of the structure during the thermal cycle. The inset in (c) is a magnified plot for the $N-T$ curve from 35.0 to $56.0^{\circ} \mathrm{C}$.

infinite, the corresponding structure is perfectly ordered and single value is resulted for column spacing in the structure. The temperature dependent orderness corresponding to the structures during the thermal cycle is shown in Figure $3 \mathrm{~b}$. It is clear that a kink occurs at $32.0^{\circ} \mathrm{C}$ in the $Q-T$ curve during the heating process. By comparing the $d-T$ and $Q-T$ curves of the heating process, it was found that the temperature at the kink in $Q-T$ curve is lower than that in $d-T$ curve. This implies that the enhancement of thermal energy during the heating process first depresses the interactions between columns, which leads to a reduction in the orderness at $32.0^{\circ} \mathrm{C}$, and then breaks the confinement of particles in columns at $35.0{ }^{\circ} \mathrm{C}$, at which point agglomerated particles start to be dispersed into the liquid.

In Figures 3a and b, the curves for the heating process vanish at $40.0^{\circ} \mathrm{C}$ because the structural pattern evolves to a disordered state. To characterize the structural evolution with increasing temperature in the disordered state, the column numbers, $N$, within a given area are counted at different temperatures. Figure 3c plots the $N-T$ curve for the structures shown in Figure 2. The plateau in the $N-T$ curve from 26.0 to $35.0{ }^{\circ} \mathrm{C}$ corresponds to the ordered structure state with fixed column spacing shown in Figure 3a. As the temperature increases above $35.0{ }^{\circ} \mathrm{C}$, a continuous reduction of $N$ with rising the temperature is obtained, no matter whether the structure is at an ordered or a disordered state. And finally at $56.0^{\circ} \mathrm{C}$, all magnetic columns disappear and hence $N$ becomes zero.

When the temperature is lowered from 56.0 to $26.0^{\circ} \mathrm{C}$, there is no column formed until the temperature approaches $54.0^{\circ} \mathrm{C}$. Hence, a non-zero value results for $N$ at $54.0{ }^{\circ} \mathrm{C}$, as shown with the inset of Figure $3 \mathrm{c}$ for the $N-T$ curve during the cooling process from $56.0^{\circ} \mathrm{C}$. When the temperature is decreased from 54.0 to $51.0^{\circ} \mathrm{C}$, more particles agglomerate to form columns so the $N$ increases, followed by a plateau in the $N-T$ curve as the temperature varies from 51.0 to $41.0{ }^{\circ} \mathrm{C}$. The plateau in 
the $N$-T curve for temperatures from 51.0 to $41.0^{\circ} \mathrm{C}$ is attributed to the phenomenon in which the existed columns attract more dispersed particles from surrounding magnetic fluid, instead of forming new column. Thus, the column number remains unchanged, but each column becomes larger with lowering temperature. Furthermore, the number of columns increases continuously with the decrease in temperature from 41.0 to $26.0{ }^{\circ} \mathrm{C}$ due to the splitting of columns, as shown in Figure 3c. It is worth noting that the structure evolves from a disordered state to an ordered pattern at $29.0{ }^{\circ} \mathrm{C}$ during the cooling process. Thus, the $d-T$ and the $Q-T$ curves can be plotted for the cooling process, as shown in Figures $3 \mathrm{a}$ and $\mathrm{b}$. The $d$ was found to be reduced, and meanwhile, a higher orderness was achieved when the temperature decreased from 29.0 to $26.0{ }^{\circ} \mathrm{C}$.

In Figure $3 \mathrm{~b}$, the $Q$ at a certain temperature during the heating process is always larger than that at the same temperature during the cooling process. This is due to the fact that a larger value of $d$ is achieved at a given temperature during the cooling process, as compared to the heating process shown in Figure 3a. Thus, the repulsive interaction among magnetic columns with parallel magnetizations in the magnetic fluid film becomes weaker for a larger $d$. Since the formation of the ordered structure of columns in the magnetic fluid film under external magnetic fields is due to the isotropic repulsive interactions among magnetic columns, the ordered structure turns to be melting when the repulsive interactions are reduced at a larger $d$. Consequently, a larger dispersion in the column spacing and a smaller $Q$ are resulted. If we plot the $d$-dependent orderness, $Q(d)$, for both the heating and the cooling processes, as shown in the inset of Figure $3 \mathrm{~b}$, the $Q$ of a given $d$ during the cooling process is clearly larger than that for the heating process. This reveals that one can promote the orderness of an ordered structure in a magnetic fluid film through a thermal cycle. Obviously, a lower temperature is resultant for the final structure after a thermal cycle to obtain the same value of $d$ as that of the initial ordered structures.

\section{Conclusion}

The ordered structure in a magnetic fluid film undergoes a process of evolution during a thermal cycle: from the initially ordered state to a disordered pattern, to mono-dispersion, and alternatively back to an ordered structure through a state of column formation/split. It is noted that the structural evolution is irreversible under a thermal cycle. In addition, the orderness of an ordered structure in a magnetic fluid film during the cooling process is significantly reduced compared to that of the heating process. However, for the same distance $d$ between columns, the orderness during the cooling process is higher than that of the heating process.

This work was supported by National Science Council of ROC under grant Nos. NSC92-2816-M-002-0005-6, NSC93-2112-M003-007, NSC93-2120-M-003-001, and NSC93-2112-M-003-017.

\section{References}

1. F. Charles, J. Hayes, Colloid Inter. Sci. 52, 239 (1975)

2. R.E. Rosensweig, J. Popplewell, Int. J. Appl. Electromagn. In Mater. 2 Suppl., 83 (1992)

3. S. Taketomi, S. Ogawa, H. Miyajima, S. Chikazumi, IEEE Trans. Magn. 4, 384 (1989)

4. Hao Wang, Yun Zhu, C. Boyd, Weili Luo, A. Cebers, R.E. Rosensweig, Phys. Rev. Lett. 72, 1929 (1994)

5. Chin-Yih Hong, H.E. Horng, I.J. Jamh, C.J. Hsu, Y.D. Yao, H.C. Yang, J. Appl. Phys. 81, 4275 (1997)

6. J.-C. Bacri, A. Cebers, R. Perzynski, Phys. Rev. Lett. 72, 2705 (1994)

7. C. Flament, G. Pacitto, J.-C. Bacri, I. Drikis, A. Cebers, Phys. Fluids 10, 2464 (1998)

8. I.J. Jang, H.E. Horng, J.M. Wu, Chin-Yih Hong, Y.C. Chiou, H.C. Yang, J. Magn. Magn. Mater. 201, 317 (1999)

9. Chin-Yih Hong, Herng-Er Horng, F.C. Kuo, S.Y. Yang, H.C. Yang, J.M. Wu, Appl. Phys. Lett. 75, 2196 (1999)

10. Herng-Er Horng, Chin-Yih Hong, Wai Bong Yeung, Hong-Chang Yang, Appl. Opt. 37, 2674 (1998)

11. Herng-Er Horng, S.Y. Yang, S.L. Lee, Chin-Yih Hong, H.C. Yang, Appl. Phys. Lett. 79, 350 (2001)

12. US Patent 5, 948, 321

13. Chin-Yih Hong, C.H. Ho, H.E. Horng, Chun-Hui Chen, S.Y. Yang, Y.P. Chiu, H.C. Yang, Magnitnaya Gidrodinaika 35, 364 (1999)

14. Herng-Er Horng, S.Y. Yang, S.L. Lee, Chin-Yih Hong, H.C. Yang, Appl. Phys. Lett. 79, 350 (2001)

15. R. Seshadri, R.M. Westervelt, Phys. Rev. B 46, 5150 (1992)

16. B.I. Halperin, D.R. Nelson, Phys. Rev. Lett. 41, 121 (1978) 